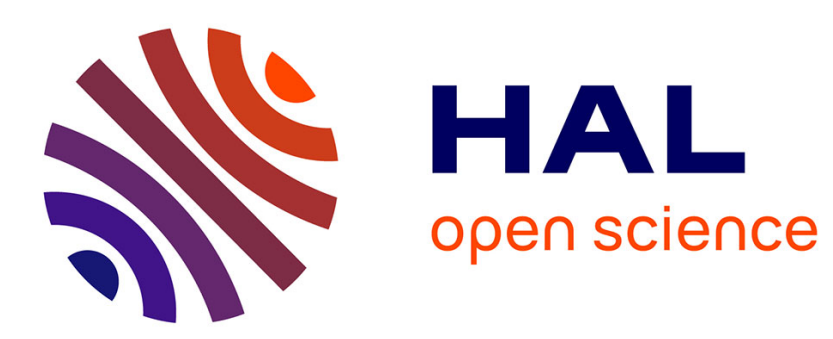

\title{
Spherical magnetic nanoparticles: magnetic structure and interparticle interaction
}

\author{
Vincent Russier
}

\section{To cite this version:}

Vincent Russier. Spherical magnetic nanoparticles: magnetic structure and interparticle interaction. Journal of Applied Physics, 2009, 105 (7), pp.073915. 10.1063/1.3093966 . hal-00366900v2

\section{HAL Id: hal-00366900 \\ https://hal.science/hal-00366900v2}

Submitted on 14 Apr 2009

HAL is a multi-disciplinary open access archive for the deposit and dissemination of scientific research documents, whether they are published or not. The documents may come from teaching and research institutions in France or abroad, or from public or private research centers.
L'archive ouverte pluridisciplinaire HAL, est destinée au dépôt et à la diffusion de documents scientifiques de niveau recherche, publiés ou non, émanant des établissements d'enseignement et de recherche français ou étrangers, des laboratoires publics ou privés. 


\title{
Spherical magnetic nanoparticles: magnetic structure and interparticle interaction
}

\author{
V. Russier * \\ ICMPE, UMR 7182 CNRS and Université Paris Est, \\ 2-8 Rue Henri Dunant, F-94320 Thiais, France.
}

\begin{abstract}
The interaction between spherical magnetic nanoparticles is investigated from micromagnetic simulations and ananlysed in terms of the leading dipolar interaction energy between magnetic dipoles. We focus mainly on the case where the particles present a vortex structure. In a first step the local magnetic structure in the isolated particle is revisited. For particles bearing a uniaxial magnetocrystaline anisotropy, it is shown that the vortex core orientation relative to the easy axis depends on both the particle size and the anisotropy constant. When the particles magnetization present a vortex structure, it is shown that the polarization of the particles by the dipolar field of the other one must be taken into account in the interaction. An analytic form is deduced for the interaction which involves the vortex core magnetization and the magnetic susceptibility which are obtained from the magnetic properties of the isolated particle.
\end{abstract}

\footnotetext{
${ }^{*}$ corresponding author russier@icmpe.cnrs.fr
} 


\section{Introduction}

With the increasing progress in the synthethis of magnetic objects of nanometric scale such as spherical nanoparticles, nanodots, nanorings or layered films the diversity of systems made of such nano-objects as building blocks either as 2D or 3D assemblies in non magnetic environment [1, 2, 3, 国 or in colloidal suspensions as ferrofluids [0, [6] is continously growing. The magnetic behavior of magnetic nanometric particles either isolated or in nanostructured bulk materials is now quite well undertood both from experiments or numerical calculations [1, 3, [] but a precise knowledge of interparticles interactions and of their influence on the macrocopic properties is still needed. Indeed, the interparticle coupling has been investigated in a variety of systems, such as nanograins [8, 9, 10, 11, 12] nanorings [13, 14, 15] or cylindrical nanodots 116, 17, 18, 19] with a predominant attention paid on short range effects, such as exchange coupling, or on the influence of the coupling between single domain particles on the global magnetic properties. Conversely the long ranged interaction still deserves attention especially in cases where the magnetic structure of the isolated particle is complex (vortex [20, 21, 22, 23, 24] or onion 25.26] states for examples). A lot of work remains to be done on this point especially for spherical particles; in particular it seems important to develop models including the long ranged and anisotropic dipolar interaction. In the simple case of single domain particles the leading term in the interaction is the long range dipolar interaction which may lead to complex structures according to the shape of the particles on the one hand and the density and the dimensionality of the whole sytstem on the other hand [27, 28, 29, 30]. In the case of particles with a non trivial internal magnetic structure, the interaction between particles is to be determined first. Indeed, it is generally admitted that when particles present a vortex structure, the resulting strong reduction of the magnetic moment at zero external field makes the dipolar interaction negligible. One aim of this work is to examine this point more precisely. In this work we focus on the interaction between spherical particles made of soft magnetic material (permalloy as an example) when they reach the vortex regime. We consider the simple situation of only two approaching spheres in a dumbell configuration. We especially compare the caculated interaction to the expected dipolar term. It is shown that two parameters characterizing the isolated particle play a central role: the magnetization of the vortex core and the suceptibility from which the polarization energy of one sphere in the dipolar field due the second one is calculated.

\section{Magnetization structure and hysteresis}

Since our purpose is to model soft magnetic particles in a general way rather than to focus on particles of a given material, the magnetic characteristics are somewhat arbitrary and correspond roughly to permalloy: the value of the exchange constant is $A_{x}=1.10^{-11} \mathrm{~J} / \mathrm{m}$, the saturation magnetization $J_{s}=1 T$ and the anisotropy is of uniaxial symmetry with a constant $K_{1}$ ranging from $K_{1}=0$ to $K_{1}=7.10^{4} \mathrm{~J} / \mathrm{m}^{3}$. The particle radius is varied from $R=10 \mathrm{~nm}$ to $45 \mathrm{~nm}$. In the following the particle volume will be denoted by $v_{s}$. We determine the magnetization structure in the framework of 
the micromagnetism formalism from the minimization of the total energy which is given by

$$
\begin{aligned}
E_{t o t} & =E_{x}+E_{a}+E_{d m}+E_{Z} \\
& =\int_{\Omega}\left[A_{x} \Sigma_{i}\left(\nabla m_{i}(\vec{r})\right)^{2}+K_{1}\left(1-(\vec{m}(\vec{r}) \cdot \hat{a})^{2}\right)-\frac{1}{2} \mu_{0} M_{s} \vec{m}(\vec{r}) \cdot \vec{H}_{d e m}-\mu_{0} M_{s} \vec{H}_{e x} \cdot \vec{m}(\vec{r})\right] d \vec{r}
\end{aligned}
$$

where $E_{x}, E_{a}, E_{d m}$ and $E_{Z}$ are the exchange, anisotropy, demagnetizing and Zeeman terms respectively, $\hat{a}$ is the unit vector in the direction of the easy axis and $\Omega$ is the total volume of magnetic material, wich can include more than one particle. $\vec{m}(\vec{r})$ is the reduced magnetization density, related to the total magnetization by $M_{t}=\int_{\Omega} M_{s} \vec{m}(\vec{r}) d \vec{r}$. The calculations are performed with the micromagnetic code MAGPAR [31] which is based upon a finite element method. The problem includes two length scales, namely the exchange length, $l_{e x}=\left(2 \mu_{0} A_{x} / J_{s}^{2}\right)^{1 / 2}$ and the Bloch wall length $l_{B}=$ $\sqrt{A_{x} / K_{1}}$. Here, given the parameters chosen we have $l_{e x}=5.013 \mathrm{~nm}$ and $l_{B}>12 \mathrm{~nm}$. The value of the dimensionless parameter, $K=2 K_{1} /\left(\mu_{0} M_{s}^{2}\right)$ as defined in [20] ranges in between 0 and 0.175 . The mesh used in the calculations is such that the largest tetaedron size is smaller than $l_{e x}$ which imposes typically a mesh with $N_{f e} \sim 10^{5}$ elements for one sphere of radius $R \simeq 40 \mathrm{~nm}$. We first calculate the magnetic structure of one isolated sphere in terms of both the size and the anisotropy constant $K_{1}$. In order to characterize the magnetic state in the particle, we consider the local magnetization profile, $\vec{m}(\mathbf{r})$, which in the vortex regime, is decomposed in its cylindrical components using the vortex axis, say $\hat{v}$, as the cylindrical axis

$$
\vec{m}(\mathbf{r})=m_{v} \hat{v}+m_{\varphi} \hat{\varphi}+m_{\rho} \hat{\rho}
$$

where $\hat{\rho}$ and $\hat{\varphi}$ are the radial and tangential unit vectors of the projection of $\mathbf{r}$ in the plane normal to $\hat{v}$. In the following, hatted letters denote unit vectors. The axis $\hat{v}$ is defined and actually determined as the mean direction of the local magnetization in the central part of the vortex, as shown in figure (1). In a first step we focus on the behavior of the magnetization $M$ in terms of the external field, $H_{e x}$, especially for the variation of the field from small values up to saturation field; however we do not focus on the nucleation field. First of all, as is well known, small particles up to a threshold value, $R_{S D}$, are uniformly magnetized in a single magnetic domain and the hysteresis curve is a square. With our set of parameters, we get $R_{S D}=18 \mathrm{~nm}$, for $K_{1}=0$ and $22 \mathrm{~nm}$ for $K_{1}=310^{4} \mathrm{~J} / \mathrm{m}^{3}$, in agreement with the result of the micromagnetic calculations of [32] and with the estimation given in [20]. Then a vortex structure is obtained, characterized by a vanishing value of the radial component $m_{\rho}(\mathbf{r})$, and $\left|m_{\varphi}(\rho)\right|$ varying from $\left|m_{\varphi}(\rho)\right|=0$ inside the vortex core, $\rho<r_{c}$, to $\left|m_{\varphi}(\rho)\right|=1$ in the vicinity of the particle surface $\rho \sim \rho_{\max }=R \sin (\theta(z))$. At zero external field, the vortex direction, $\hat{v}$ is arbitrary when $K_{1}=0$, while for $K_{1} \neq 0$, the direction taken by $\hat{v}$ relative to the easy axis $\hat{a}$ is controlled by the anisotropy energy which tends to allign $\vec{m}(\vec{r})$ on $\hat{a}$. The anisotropy energy depends on both the value of $K_{1}$ and the volume fraction of the particle where $\hat{m}(\vec{r})$ is oriented parallel or antiparallel to $\hat{a}(|\hat{m} . \hat{a}| \simeq 1)$. The ratio of the volume fraction corresponding to the vortex core $v_{c}$, characterized by $\hat{m}$ oriented parallel to $\hat{v}$, to the volume fraction where $\vec{m}(\vec{r})$ is oriented normal to $\hat{v}$ is directly related to the volume of the particle, $v_{s}$. Roughly speaking the ratio of the total volume where $|\hat{m} . \hat{a}| \simeq 1$ is 
either $v_{c} / v_{s}$ or $(1 / 2)\left(v_{s}-v_{c}\right) / v_{s}$ if $\hat{v}$ is parallel or normal to $\hat{a}$ respectively. According to this scheme the stability condition for the vortex direction $\hat{v}$ to be normal to $\hat{a}$ reads $v_{c}<(1 / 3) v_{s}$. We can refine this very crude determination of the thershold by introducing the magnetization profile and imposing in (2) $m_{\rho}=0$. Then $m_{\varphi}=\sqrt{1-m_{v}^{2}}$ and we get

$$
\begin{array}{r}
E_{a}^{/ /}=-K_{1} \int_{-R}^{R} d z \int_{0}^{R(z)} m_{v}(\rho)^{2} 2 \pi \rho d \rho \\
E_{a}^{\perp}=-K_{1} \int_{-R}^{R} d z \int_{0}^{R(z)} \frac{1}{2}\left(1-m_{v}(\rho)^{2}\right) 2 \pi \rho d \rho
\end{array}
$$

for $\hat{v}$ parallel or normal to $\hat{a}$ respectively. Then we assume that the component $m_{v}(\rho)$ depends on $\rho$ only through $r^{*}=\rho / r_{s c}, r_{s c}$ being the pertinent scaling length (either $l_{e x}$ for $K_{1}=0$ or a function of both $l_{e x}$ and $l_{B}$ otherwise) and we neglect its dependence with respect to $z$. Hence, exploiting $m_{v}\left(\rho>r_{c}\right)=0$, we set the upper bound in the integral over $m_{v}$ to $\infty$ and we write $\left(E_{a}^{\perp}-E_{a}^{/ /}\right)$in the form

$$
E_{a}^{\perp}-E_{a}^{/ /}=-K_{1}\left(\frac{1}{2} v_{s}-3 R r_{s c}^{2} I\right) \quad \text { with } \quad I=\int_{0}^{\infty} m_{v}\left(r^{*}\right)^{2} 2 \pi r^{*} d r^{*}
$$

The stability condition for a vortex normal to $\hat{a}$ is now

$$
\frac{R}{r_{s c}}>\sqrt{\frac{9 I}{2 \pi}}
$$

which must be read as $R>r_{s c} \sqrt{9 I /(2 \pi)}=R_{t h}\left(K_{1}\right)$ when $R$ is varied at constant $K_{1}$, or conversely as $r_{s c}<R \sqrt{2 \pi /(9 I)}$ when the role of the magnetic characteristics is investigated for a given particle size. Equation (5) can be rewritten in a more convenient form for the practical calculatio $J / m^{3} \mathrm{~ns}$ of $I$, namely : $S^{*}=\sqrt{9 I^{*}\left(r_{s c}\right) /(2 \pi)}<1$ where $I^{*}$ is given by (4) with $r^{*}$ replaced by $(r / R)$ and the upper bound replaced by $(r / R)_{\max }=1$. Of course $I^{*}$ is then dependent on the value of $r_{s c}$ which is emphasized by the notation $I^{*}\left(r_{s c}\right)$. In any case, such an estimation is not supposed to provide an accurate determination of the threshold value of either $R$ or $r_{s c}$ for the orientation of $\hat{v}$ normal to $\hat{a}$ but to predict at a qualitative level the effect of either the particle size or the magnetic parameters on the direction taken by the vortex. We can scale the vortex radius, $r_{c}$ on the smallest of the two characteristic lengths, $r_{s c}=\inf \left(l_{e x}, l_{B}\right)$; however, this scaling may be taken with care and instead we can consider, when $l_{B}$ increases, a scaling radius in the form of a function $r_{s c}\left(l_{e x}, l_{B}\right)$. Notice that when using $S^{*}$ instead of $S$ one has not to explicit the dependence of $r_{s c}$. Therefore, at both $A_{x}$ and $J_{s}$ kept constant, we deduce from equ. (5) that $\hat{v}$ gets $\perp \hat{a}$ when the sphere radius is increased at $K_{1}$ constant or when $K_{1}$ is increased at $R$ constant. As we shall see in the following the stability condition for the orientation of $\hat{v}$ relative to $\hat{a}$ agrees with this qualitative conclusion. Notice that the orientation of the vortex relative to the axis of easy magnetization is also found to be size dependent in the case of the cubic anisotropy [32] : in this latter case, the vortex is parallel to the axis of easy magnetization in large spheres.

The orientation of the vortex relative to $\hat{a}$ can be determined from the magnetization profile, $\hat{m}(\vec{r})$ 
as well as from the magnetization curve in terms of the external field, $M\left(H_{e x}\right)$ by chosing the direction of the external field, $\hat{h}_{e x}$ either parallel or normal to $\hat{a}$. Indeed, we expect the magnetization process to differ according to the direction of the external field relative to the vortex one. We keep in mind the well known behavior of the vortex in the flat cylindrical nanodots where the magnetization is found to result from the shift of the vortex core when the field is applied normal to the vortex direction. Here, in the case of $\hat{v}\left(H_{e x}=0\right) \perp \hat{a}$ we expect a similar behavior for small values of the external field when $\hat{h}_{e x}=\hat{a}$, up to the rotation of the vortex core along the direction of the field for high values of $H_{e x}$ before the magnetization in the whole volume of the sphere becomes oriented along $\hat{h}_{e x}$. Morever, in this case, we expect to have no remanence in the direction of the field, since in the vicinity of $H_{e x}=0$ there is no net magnetization normal to the vortex direction. On the other hand a non vanishing magnetization at $H_{e x}=0$, corresponding to the vortex polarization in the direction $\hat{v} \perp \hat{a}=$ $\hat{h}_{e x}$ will be obtained. Conversely, the magnetization curve corresponding to $\hat{h}_{e x} \perp \hat{a}$ still for a sphere characterized by $\hat{v}\left(H_{e x}=0\right) \perp \hat{a}$ will present the more usual shape of a loop located in between $\pm H_{c}$ with a non zero remanence corresponding to the vortex polarization.

Then we focus on the external field induced magnetization in the spherical particle. As is generally obtained in nanodots or spherical soft magnetic particles [33, 35, 36], the magnetization $M$ in the direction of the external field is found to vary nearly linearly with respect to the field, at least in the vicinity of $H_{e x}=0$ and of course away from switching points where the vortex reverses as a whole. Such a linear behavior is observed both when $\hat{h}_{e x}=\hat{v}$ or $\hat{h}_{e x} \perp \hat{v}$. (or equivalently $\hat{h}_{e x} \perp \hat{a}$ or $\hat{h}_{e x}=$ $\hat{a}$ when $R>R_{t h}\left(K_{1}\right)$ ). This means that the susceptibility $\chi$ defined as

$$
\frac{\partial M}{\partial H_{e x}}=\chi
$$

does not depend on the value of the field to a very good approximation. We emphasize that the suceptibility is well defined for particles in the vortex regime since no multidomain state occurs and therefore a demagnetized state at $H_{e x}=0$ can be ruled out. Notice that the value of $\chi$ depends on the direction of the field as will be discussed below, and we should distinguish $\chi_{\|}$from $\chi_{\perp}$ according to the direction of the field relative to $\hat{v}$. When such a distinction is not necessary it will be omitted to lighten the notations and $\chi$ is to be understood as its value corresponding to the orientation chosen for the field. Only in the case of an external field direction $\hat{h}_{e x}$ neither parallel nor normal to the vortex direction the consideration of the two values of $\chi$ is necessary. The independence of $\chi$ with respect to $H_{e x}$ can be exploited for obtaining the variation of the total energy with respect to the external field. We consider the variation of $M$ starting from $H_{e x}=0$ to a value of $H_{e x}$ such that no switching of the magnetization occurs up to $H_{e x}$ and we analyse the corresponding variation of the magnetization, $\Delta M$ as the polarization of the sphere induced by the field. We have $\Delta M\left(H_{e x}\right)=\chi H_{e x}$. On the other hand, we can deduce $\Delta M\left(H_{e x}\right)$ from the energy, $E\left(H_{e x}\right)$ by writting an equilibrium equation

$$
\frac{\partial E_{t o t}(\Delta M)}{\partial \Delta M}=0
$$

which determine the equilibrium value of $\Delta M$. The total energy depends explicitly on $H_{e x}$ through 
the Zeeman term, $-\mu_{0} H_{e x}\left(m(0) \hat{v} . \hat{h}_{e x}+\Delta M\right)$, where we have expressed the permanent magnetization in the absence of the field as $\vec{M}\left(H_{e x}=0\right)=m(0) \hat{v}, m(0)$ being the magnitude of the vortex core magnetization in the absence of the field. Then from (7) we get

$$
\frac{\partial}{\partial \Delta M}\left(E_{d m}+E_{x}+E_{a}\right)=\mu_{0} H_{e x}
$$

Therefore we get the variation of the total energy in the form

$$
\begin{aligned}
E=E\left(H_{e x}=0\right)+ & \int_{0}^{\Delta M} \mu_{0} H_{e x}\left(\Delta M^{\prime}\right) d \Delta M^{\prime}-\mu_{0} H_{e x}\left(m(0) \hat{v} \cdot \hat{h}_{e x}+\Delta M\right) \\
& =E\left(H_{e x}=0\right)+\mu_{0} \frac{\Delta M^{2}}{2 \chi}-\mu_{0} H_{e x}\left(m(0) \hat{v} . \hat{h}_{e x}+\Delta M\right)
\end{aligned}
$$

where we have used $\Delta M\left(H_{e x}\right)=\chi H_{e x}$. Notice that both (8) and (9) are exact equations while (10) holds only in the case of a linear dependence of $\Delta M\left(H_{e x}\right)$ with respect to $H_{e x}$. The second term of the r.h.s. of (9) or (10) has a simple interpretation: it is the energy of polarization of the sphere and corresponds to the energy cost of the reorientation of the magnetization inside the sphere. Equ. (10) is to be compared to the expression of the energy density of an array of coupled dots presenting a vortex structure obtained in 34; more precisely the polarization energy coincides with the second term of equ.(5) of Ref. [34] where the induced magnetization in the dot is related to the vortex shift, $s$. The polarization energy can be written equivalently as $\mu_{0}\left(\Delta M H_{e x}\right) / 2$ where $\Delta M$ is to be understood as the induced moment due to the external field. Finaly, $\chi$ can be related to the variation of the energy minus the Zeeman term

$$
\chi=\frac{1}{\mu_{0} H_{e x}} \frac{\partial\left(E_{t o t}-E_{Z}\right)}{\partial H_{e x}}
$$

\section{$3 \quad$ Interaction energy between magnetic spheres}

Now we focus on the determination of the interaction energy between two magnetic nanoparticles in terms of the interparticle distance, $r_{12}$. The interaction energy is defined in a usual way

$$
E_{\text {int }}(1,2)=E_{\text {tot }}(1,2)-E_{\text {tot }}\left(r_{12} \rightarrow \infty\right)
$$

where $E_{\text {tot }}$ denotes the total energy of the two particles system, and $(1,2)$ is a short notation for the orientation and location variables of the particles when they are brought together. We expect to get a form dictated by the dipolar interaction between the magnetic moment of the approaching spheres which reads

$$
\begin{array}{r}
E_{d i p}=\frac{\mu_{0} m_{1} m_{2}}{4 \pi r_{12}^{3}} d_{112}\left(\hat{m}_{1}, \hat{m}_{2}, \hat{r}_{12}\right) \\
d_{112}\left(\hat{m}_{1}, \hat{m}_{2}, \hat{r}_{12}\right)=\hat{m}_{1} \cdot \hat{m}_{2}-3\left(\hat{m}_{1} \cdot \hat{r}_{12}\right)\left(\hat{m}_{2} \cdot \hat{r}_{12}\right)
\end{array}
$$


where $m_{i}$ are the magnitude of the magnetic moments, $\hat{m}_{i}$ and $\hat{r}_{12}$ the unit vectors in the direction of both moments and of the vector joigning the two particles and $d_{112}$ is the angular function characteristic of the dipolar intercation. In the case of single domain particles $m_{i}=M_{s} v_{s}$ where $M_{s}$ is the saturation magnetization of the particles, and the orientations $\hat{m}_{i}$ result from the minimum of $d_{112}$ given in (14). For particles without magnetocristalline anisotropy, this gives obviously : $\hat{m}_{1}=\hat{m}_{2}=\hat{r}_{12}$ and $d_{112}=$ -2. On the other hand, if the magnetocristalline energy is non zero on both particles with easy axes $\hat{a}_{i}$, the orientations $\hat{m}_{i}$ will result of the interplay between the anisotropy energy tending to align $\hat{m}_{i}$ on $\hat{a}_{i}$ and the energy (13) tending to minimize the angular function (14). Furthermore if $K_{1}$ takes a non vanishing value only in one particle say $i=1$ and if this value is large enough to impose $\hat{m}_{1}=\hat{a}_{1}, \hat{m}_{2}$ must orient in the dipolar field due to particle 1 i.e. in such a way that $d_{112}=\hat{m}_{2} \cdot \hat{a}_{1}-3\left(\hat{m}_{2} \cdot \hat{r}_{12}\right)\left(\hat{a}_{1} . \hat{r}_{12}\right)$ is minimum.

Now we consider the case of particles large enough to present a vortex structure. In this case, the orientations of the effective moments of the particles are the vortex directions, $\hat{v}_{i}$, and the values of the moments are no more equal to $M_{s} v_{s}$ but correspond to the vortex cores magnetizations and can be obtained from the magnetization curves $M\left(H_{e x}\right)$. Let us introduce the coefficients $\alpha_{i}=m_{i} /\left(M_{s} v_{s}\right)$ (in the following, we shall only consider the case of identical particles, so we drop the index $i$ ). $\alpha$ depends of course on the location of the second particle, which will be denoted in short by $\alpha\left(r_{12}, d_{112}\right)$ or $\alpha(1,2)$. The value taken by $\alpha$ is not trivial since on the one hand it must be determined from the characteristics of the isolated particle and from the polarization of the particle by the dipolar field of the second one. A simple approximation for the interaction energy can be built in the framework of the dipolar approximation by considering that each particle is in the dipolar field of the other one. Then, we have to take into account two contributions. The first one which corresponds to (13), is nothing but $-m_{1} H_{d i p}\left(r_{12}\right)\left(\hat{v}_{1} . \hat{h}_{d i p}(2,1)\right)$ where $H_{d i p}\left(r_{12}\right) \hat{h}_{d i p}(2,1)$ is the dipolar field created at $\mathbf{r}_{1}$ by particle at $\mathbf{r}_{2}$ and the second one is twice the polarization energy of each sphere in the field of the second one. The second contribution has been introduced in (10) for one particle in a constant external field. In the present case, the role of $m(o)$ is played by $M_{s} v_{s} \alpha(\infty)$ while the induced moment in the direction of the dipolar field is

$$
\vec{p}=p \hat{h}_{d i p}=\chi H_{d i p}\left(r_{12}\right) \hat{h}_{d i p}
$$

We first consider the case where the vortex $\hat{v}_{i}$ is free to orient in the direction of the dipolar field due to particle $j \neq i$. This is the most general case since it corresponds to both the absence of anisotropy or particle large enough for the vortex to be normal to the easy axis. In this case we have

$$
p \hat{h}_{d i p}=\left(\alpha\left(r_{12}, d_{112}\right)-\alpha(\infty)\right) M_{s} v_{s} \hat{h}_{d i p}=\Delta \alpha\left(r_{12}, d_{112}\right) M_{s} v_{s} \hat{v}
$$

Now adding twice the second term of $(10)$ to the total dipolar energy we get for the interaction energy

$$
E_{\text {int }}(1,2)=\frac{\mu_{0}\left(M_{s} v_{s}\right)^{2}}{4 \pi r_{12}^{3}} \alpha(\infty)\left(\alpha(\infty)+\Delta \alpha\left(r_{12}, d_{112}\right)\right) d_{112}\left(\hat{m}_{1}, \hat{m}_{2}, \hat{r}_{12}\right)
$$


which coincides with the interaction energy between polar polarizable hard spheres [37. This is the important result of this section. It relates the interaction energy to the magnetic charateristics of the isolated spheres, namely, $\alpha(\infty)$ and $\chi$ through $\Delta \alpha$. Notice that this form for the interaction energy should hold not only in the case of two particles but also more generaly for an assembly of particles. In the latter case, the solvation of the total dipolar field and thus the determination of $\Delta \alpha\left(r_{12}, d_{112}\right)$ becomes a difficult task. In the simple case of two particles, introducing $u=\chi /\left(4 \pi R^{3}\right)\left(=\chi^{*} / 3\right.$ where $\chi^{*}=\chi / v_{s}$ is the reduced susceptibility) we get

$$
\Delta \alpha=\frac{-u \alpha(\infty) d_{112}}{\left(\left(r_{12} / R\right)^{3}+u d_{112}\right)}
$$

\section{Results}

We analyse first the magnetic behavior of the isolated particle with a special attention paid on the characterization of the vortex structure at low external fields. The magnetization curve is displayed in figure (2) for $K_{1}=0$ and $R=45 \mathrm{~nm}$ and the corresponding magnetic structure, through the local magnetic moment components (2), is shown in figures 3 and 1 for the remanent state and in the vicinity of the coercive field, before and after the reversal of the vortex core. Since $K_{1}=0$, the vortex direction, $\hat{v}$ coincides with the direction of the external field. These results put in evidence the vortex structure and in particular the vortex core is reversed as a whole at the coercive field, with a nearly frozen $\vec{m}(\vec{r})$ structure. Moreover, we find that the reversal of $\vec{m}(\vec{r})$ results from a global rotation since the component $m_{\varphi}$ changes sign. When $K_{1} \neq 0$ as described at the qualitative level in section (2) $\hat{v}$ is parallel to the easy direction $\hat{a}$ for small values of $R$, and becomes normal to $\hat{a}$ beyond a $K_{1}$ dependent threshold value $R_{t h}\left(K_{1}\right)$. When $\hat{a} \perp \hat{v}$, the vortex core is free to rotate in the plane normal to $\hat{a}$ and therefore will orient parallel to the external field if $\hat{h}_{e x} \perp \hat{a}$. As an example, we show in figure (5) the magnetization curve for the two directions of the external field $\hat{h}_{e x}=\hat{a}$ and $\hat{h}_{e x} \perp \hat{a}$, in the case $R=$ $45 \mathrm{~nm}$. Moreover, in the former case, the magnetization parallel and normal to the external field, $M_{\|}$ and $M_{\perp}$ are displayed. The magnetization behavior in terms of the external field corresponds to the situation $\hat{v} \perp \hat{a}$; indeed, the remanence vanishes when $\hat{h}_{e x} \| \hat{a}$, while $M_{\perp}$ takes a nearly constant value when $H_{e x}$ is varied in the central part of the $M_{\perp}\left(H_{e x}\right)$ curve. Moreover this value coincides with the

remanence obtained for $\hat{h}_{e x} \perp \hat{a}$ or equivalently $\hat{h}_{e x} \| \hat{v}$ and therefore corresponds to the vortex core magnetization. The independence of $M_{\perp}$ with respect to $H_{e x}$ in the central part of the $M_{\perp}\left(H_{e x}\right)$ curve shows that the variation of the magnetization $M_{\|}\left(H_{e x}\right)$, i.e. in the direction normal to $\hat{v}$, corresponds to a shift of the vortex core normal to the direction of the field. The vortex core magnetization is thus nearly constant and given by the value of $M_{\perp}$ in that part of the curve. This is in agreement with the magnetization process obtained in the flat nanodot vortex structures. Finally $M_{\perp}$ sharply vanishes when the vortex rotates in the direction of the field, where the magnetization curve $M_{\|}$whith $\hat{h}_{e x}=\hat{a}$ presents the hysteretic wings, similar also to what is found in the flat nanodot case where however this last value of the field corresponds to the vortex anhihilation prior to the saturation of the dot. The behavior of $M\left(H_{e x}\right)$ outlined above is coroborated by the evolution with the value of the field of the structure of $\hat{m}(\vec{r})$, shown on figure (6), where we see that the linear variation of $M_{\|}\left(H_{e x}\right)$ 
in the central part of the curve can be associated to a shift of the vortex in a direction normal to $\hat{m}$. From the evolution of $M_{\|}$for $\hat{h}_{e x}=\hat{a}$ with the particle size, displayed on figure (7), one can determine the threshold value $R_{t h}\left(K_{1}\right)$ beyond which $\hat{v}$ is normal to $\hat{a}$. Here we find $R_{t h} \simeq 28 n m$ for $K_{1}=3.10^{4} \mathrm{~J} / \mathrm{m}^{3}$ (strictly speaking, $26 \mathrm{~nm} \leq R_{t h} \leq 30 \mathrm{~nm}$ ). We have calculated numerically the integral $I^{*}\left(r_{s c}\right)$ defined after equation (4) from which we find that the threshold condition (5) is satisfied (see table $\mathbb{Q}$ ) in good agreement with the onset of the vortex structure deduced from the magnetization. Indeed, from this calculation, we get $S^{*}=1$ for $R=26.5 \mathrm{~nm}$ when $K_{1}=3.10^{4} \mathrm{~J} / \mathrm{m}^{3}$ and thus $R_{t h}=26.5 \mathrm{~nm}$ in agreement with the value deduced from the behavior of the magnetization $M\left(H_{e x}\right)$. Similarly by decreasing $K_{1}$ at constant $R=45 \mathrm{~nm}$, we find that the range of external field where the vortex is normal to $\hat{a}$ is reduced and then vanishes for $K_{1}=2.10^{3} \mathrm{~J} / \mathrm{m}^{3}$. Therefore we confirm our prediction that $\hat{v} \perp \hat{a}$ for $K_{1}>K_{1 t h}(R)$ at constant $R$. Then the value of $\chi$ is determined from the slope of the magnetization curve, $M\left(H_{e x}\right)$ in terms of $H_{e x}$. The results are listed in table 1 . We also check that equ. (11) is satisfied (see table [1).

\subsection{Interaction beween particles}

We first consider the case of monodomain particles; for this we chose $R=10 \mathrm{~nm}$. As expected the interaction energy is exactly given by the dipolar term with $m_{i}=M_{s} v_{s}$. When $K_{1}=0$ for both particles, the energy minimization leads to $d_{112}(1,2)=-2$ and we thus mainly test the $1 / r_{12}^{3}$ dependence of the interaction. On the other hand, we have also considered the case where only one particle bears a non vanishing uniaxial anisotropy with a value of $K_{1}$ large enough to impose the orientation of its moment, $\hat{m}$ parralel to the easy axis $\hat{a}$. Then the moment of the second particle orient itself in the field of the fixed particle in order to minimise the angular function $d_{112}$. This provide an additionnal test of the behavior of the interaction through its angular dependence. The results are displayed in table III. Now we consider the vortex regime with particles of radius $R=35 \mathrm{~nm}$ or $R=45 \mathrm{~nm}$. We start from particles without anisotropy, $K_{1}=0$. In this case only one value for the susceptibility, $\chi_{\|}$, is to be considered, since the vortex allign spontaneously in the direction of the dipolar field. The two parameters involved in the expression of the interaction, $\chi$ and $\alpha(\infty)$, are determined first from the magnetization curve of the isolated particle. As a first test, we look at the angular dependence of the interaction energy. To this aim we start from the two spheres at a large distance and we minimize the total energy corresponding to non interacting spheres. Then we decrease the distance $r_{12}$ down to a not too small value of the ratio $r_{12} / R$ and we perform a rotation of one sphere, say 2 , arround the other one which is kept fixed. In this first calculation, we just calculate the components of the energy without minimization; we thus obtain the energy at a fixed value of local magnetic structure in the spheres, disregarding the polarization energy. The result is displayed in figure (8) in the case $r_{12} / R$ $=4$ and different values of the angular function $d_{112}$ calculated by using $\hat{m}_{i}=\hat{v}_{i}$. We clearly obtain a linear dependence of $E_{\text {int }}(1,2)$ in terms of $d_{112}(1,2)$, and moreover the proportionality factor is exactly the result of the dipolar interaction, as deduced from (13). We thus conclude that when the structure 
inside the spheres is frozen, the resulting interaction energy is indeed given by the dipolar interaction between the vortex cores. Then we consider the interaction energy after relaxation of the structure in the spheres, namely from the result of the total energy minimization in terms of the distance between particles. As expected and in agreement with eq. (17) the value we get for $d_{112}(1,2)$ is very close to $d_{112}=-2$ especially for short distances. The result for the interaction is shown in figure (9). We also compare the result corresponding to the dipolar interaction including the polarization energy or without this las term. This later approximation amounts to model the interaction by that between the dipoles corresponding to the isolated particles vortex cores. The approximation introduced in (17) is in very good agreement with the calculated result, for distances down to $r_{12} / R \sim 2.75$, and the agreement for $r_{12} / R=2.5$ is still fairly good. Moreover we see that the inclusion of the polarization energy is quite important; indeed, the dipolar interaction calculated with the moments resulting from the isolated particles vortex cores reproduces the interaction only for distances larger than $3.35 R$.

In the case of particles with non zero uniaxial anisotropy, we focus on a situation where the vortex direction, $\hat{v}$, is normal to the easy axis at zero external field. As an example we choose $K_{1}=3.10^{4} \mathrm{~J} / \mathrm{m}^{3}$ and either $R=45 \mathrm{~nm}$ or $R=35 \mathrm{~nm}$. One can impose the plane in which the vortex is free to rotate via the direction chosen for the easy axis. Here we consider two situations where the two particles have the same easy axis, say $\hat{a}=\hat{z}$ and the unit vector joigning the particles $\hat{r}_{12}$ is either normal or parallel to $\hat{a}$. Thus the equilibrium configuration of the particles corresponds to $\hat{v}_{1}=\hat{v}_{2}=\hat{r}_{12}$ and $d_{112}(1,2)=-2$ in the former case and $\hat{v}_{1}=-\hat{v}_{2} \perp \hat{r}_{12}$ and $d_{112}(1,2)=-1$ in the latter case. The results are summarized in figure (10) where we plot the interaction energy normalized by the value at the shortest distance considered, $r_{12}=2.25 R$. The interaction energy is still very close to the dipolar plus polarization energy, eq (17) when $d_{112}=-1$, while in the case where the vortices are in line, the agreement for short distances is more qualitative. This is mainly due to an underestimation of the induced polarization by the dipolar field. We are lead to this conclusion by fitting the values of the parameters $\alpha$ and $u$ in order to reproduce the calculated interaction energy by equ. (17). Doing this we can reproduce the calculated interaction energy only by using a non negligible enhancement of $u$ while the fitted value of $\alpha$ remains very close to that calculated on the isolated sphere. The fitted results are also displayed in figure (10). To get a similar agreement with what is obtained in the case $K_{1}=0$ with $d_{112}=-2$, the fitted value of $u$ and $\alpha$ are $1.25 u^{\text {calc }}, 1.03 \alpha^{\text {calc }}$ and $1.45 u^{\text {calc }}, 1.05 \alpha^{\text {calc }}$ for $R=35 \mathrm{~nm}$ and $45 \mathrm{~nm}$ respectively.

The results of this work are twofold. First we have precised the local magnetic structure in the sphere, and shown that beyond the well documented single domain to vortex transition in the case of a uniaxial anisotropy the vortex direction is normal to the easy axis once the particle radius is larger than a threshold value, $R_{t h}\left(K_{1}\right)$ for which a simple estimation is given. Then the interaction between particles is shown to present a dipolar character depending on two parameters characterising the isolated particle, namely the vortex core magnetization and the suceptibility. The vortex core magnetization is strongly reduced when compared to the saturation magnetization $M_{s}$ which is quantified by the parameter $\alpha \sim 0.2$ and this makes the interaction rather small but nevertheless non negligible. The order of magnitude of the interaction energy at distance $r_{12}=2.25 R$ is slighltly 
smaller than the barrier necessary to reverse the vortex core. However due to both its long range and its anisotropy the dipolar interaction is likely to lead to measurable effects in experimental assemblies of such particles. On a qualitative point of view, we do think that some of the finding of 24] are in agreement with the manifestation of dipolar effects, namely the tendency to form chains and to allign the vortex cores. In ref ( [24]) a micromagnetic simulation was already performed and was in agreement with the experiments; however, here we go a step forward by clearly pointing the dipolar character of the interaction between spherical nanoparticles. This allows us to predict that in a general way the behavior of dipolar and polarizable hard spheres will be transferable to assemblies of such particles even in the vortex regime. In this field, a very rich panel of structures is expected for both 2D systems [27] (and reference therein), 28, 29] and 3D systems [30].

\section{Acknowledgements}

The author acknowledges stimulating and important discussions with Dr. Y. Champion (ICMPE, CNRS, Thiais France), Dr. F. Mazaleyrat (SATIE, ENS Cachan France) and Pr. L. Bessais (ICMPE, CNRS, Thiais France). 


\section{References}

[1] R. Skomski, J. Phys. Condens. Matter 15 (2003), R841.

[2] J.I. Martín, J. Nogués, K. Liu, J.L. Vincent and I.K. Schuller, J. Magn. Magn. Mater. 256 (2003), 449.

[3] S.D. Bader, Rev. Modern Phys. 78 (2006), 1.

[4] S.H.Sun and C.B. Murray, J. Appl. Phys. 85 (1999), 4325.

[5] V. Cabuil, Curr. Opin. Colloid Interface Sci. 5 (2003), 44.

[6] Holms and J.J. Weis, Curr. Opin. Colloid Interface Sci. 10 (2005) 133.

[7] R.C. O'Handley, Modern magnetic materials. Principles and applications, Wiley intersicence NeyYork (2000).

[8] T. Schrefl, T. Fidler and H. Kronmuller, Phys. Rev. B 49 (1994), 6100.

[9] M.K. Griffiths, J.E.L. Bishop, T.W. Tucker and H.A. Davis, J. Magn. Magn. Matter., 234 (2001), 331.

[10] C.B. Rong et al J. Magn. Magn. Mater. 302 (2006), 126.

[11] T. Schrefl et al., J. Magn. Magn. Mater. 207 (1999), 45.

[12] D. Suess, Appl. Phys. Letters 89 (2006), 113105.

[13] T. Miyawaki, K. Toyoda, M. Kohda A. Fujita and J. Nitta, Appl. Phys. Letters 89 (2006), 122508.

[14] Wand, Adeye, Appl. Phys. Lett. 87 (2005), 262508.

[15] Chang et al. J. Appl. Phys. 103 (2008), 122508.

[16] K.YU Guslienko, Appl. Phys. Lett. 75 (1999), 394.

[17] M.A. Perry, T.J. Flack, D.K. Koltsov and M.E. Welland, J. Magn. Magn. Mater., 314 (2007), 75.

[18] C.A. Ross, Phys. Rev. B 65 (2002), 144417.

[19] K.S. Buchanan, K. Yu. Guslienko, A. Doran, A. Scholl, S.D. Bader and V. Novosad, Phys. Rev. B 72 (2005), 134415.

[20] G. Bertotti, Hysteresis in magnetism, (2005), Academic Press San Diego.

[21] M. Hehn, K. Ounadjela, J.P. Bucher, F. Rousseaux, D. Decanini, B. Bartenlian and C. Chappert, Science 272 (1996), 1781.

[22] R.P. Cowburn, D.K. Koltsov and M.E. Welland, Phys. Rev. Lett. 83 (1999), 1042.

[23] T. Pokhil, D. Song and J. Nowak, J. Appl. Phys. 87 (2000), 6319.

[24] M.J. Hytch, R.E. Dunin-Borkowski, M.R. Scheinfein, J. Moulin, C. Duhamel, F. Mazaleyrat and Y. Champion, Phys. rev. Lett. 91 (2003), 257207.

[25] J. Rothman, M. Klaui, L. Lopez-Diaz, C.A.F. Vaz, A. Bleloch, J.A.C. Bland, Z. Cui and R. Speaks, Phys. Rev. Lett. 86, (2001), 1098.

[26] S.P. Li, D. PEyrade, M. NAtali, A. Lebib and Y. Chen, Phys. Rev. Lett. 86, (2001), 1102.

[27] J.J. Weis, J. Phys.: Condens. Matter 15 (2003), S1471.

[28] R.A. Trasca and S.H.L. Klapp, J. Chem. Phys. 129 (2008), 084702.

[29] V. Russier, J. Appl. Phys. bf 89 (2001), 1287.

[30] D.Kechrakos and K.N. Trohidou, Phys. Rev. B 58 (1998), 12169.

[31] W. Scholz, J. Fidler, T. Schrefl, D. Suess, R. Dittrich, H. Forster, V. Tsiantos, Comp. Mat. Sci. 28 (2003) 366-383.

http://magnet.atp.tuwien.ac.at/scholz/magpar/

[32] A. Kakay and L.K. Varga, J. Apll. Phys. 97 (2005), 083901.

[33] K. Yu. Guslienko, Appl. Phys. Letters 78 (2001), 3848. 
[34] V. Novosad, K.Yu. Guslienko, H. Shima, Y. Otani, S.G. Kim, K. Fukamichi, N. Kikuchi, O. Kitami and Y. Shimada, Phys. Rev. B 65, 060402 (2006).

[35] A. Aharoni, J. Appl. Phys. 52 (1981), 933; 55 (1983), 1049.

[36] J. Moulin and F. Mazaleyrat, J. Magn. Magn. Mater., 290-291 (2005), 540.

[37] C.J.F. Bottcher, Theory of electric polarization, Vol.1 (1973) Elsevier. 
Table I: Value of $S^{*}=\sqrt{9 I^{*}\left(r_{s c} /(2 \pi)\right.}$ involved in the stability condition (5). $K_{1}=3 \cdot 10^{4} \mathrm{~J} / \mathrm{m}^{3} \cdot I^{*}$ is defined by equ. (幽 with $r_{s c}=R$.

\begin{tabular}{|l|llll|}
\hline$R(n m)$ & 26 & 30 & 37 & 45 \\
$S^{*}$ & 1.042 & 0.685 & 0.596 & 0.533 \\
\hline
\end{tabular}

Table II: Reduced magnetic susceptibility calculated from $(a)$ : equation (6); (b) equation (11).

\begin{tabular}{|llllll|}
\hline$K_{1}$ & $R$ & $\chi_{\|}^{*(a)}$ & $\chi_{\|}^{*(b)}$ & $\chi_{\perp}^{*(a)}$ & $\chi_{\perp}^{*}{ }^{(b)}$ \\
\hline 0 & 45 & 3.229 & 3.21 & & \\
0 & 40 & 3.288 & 3.28 & & \\
0 & 37 & 3.336 & 3.33 & & \\
0 & 35 & 3.352 & 3.37 & & \\
$3.10^{4}$ & 45 & 2.887 & 2.94 & 4.589 & 4.58 \\
$3.10^{4}$ & 35 & 2.990 & & 6.510 & 6.676 \\
\hline
\end{tabular}

Table III: Angular dependence of the interaction between modomain particles. $R=10 \mathrm{~nm} ; K_{1}(1)=$ $7.10^{5} \mathrm{~J} / \mathrm{m}^{3} K_{1}(2)=0 . \Theta\left(\hat{a}_{1}\right), \Theta_{1}$ and $\Theta_{2}$ denote the angles $\left(\hat{a_{1}}, \hat{z}\right),\left(\hat{m}_{1}, \hat{z}\right)$ and $\left(\hat{m}_{2}, \hat{z}\right)$ respectively. $r_{12}=4 R . d_{112}^{(\min )}$ is the minimum value of the angular function $d_{112}$ corresponding to $\Theta_{1}$ fixed and $d_{112}^{(c a l c)}$ is the result of the numeriacal calculation. $E_{\text {int }}$ is the interaction energy per unit volume. According to the dipolar interaction the theoretical value for $E_{\text {int }} / d_{112}$ is $2072 \mathrm{~J} / \mathrm{m}^{3}$.

\begin{tabular}{|lllllc|}
\hline$\Theta\left(\hat{a}_{1}\right)$ & $\Theta_{1}$ & $\Theta_{2}$ & $d_{112}^{(\text {calc })}$ & $d_{112}^{(\text {min })}$ & $E_{\text {int }} / d_{112}\left(\mathrm{~J} / \mathrm{m}^{3}\right)$ \\
\hline$\pi / 8$ & $\pi / 7.948$ & $\pi / 4.442$ & -1.2019 & -1.202 & 2012 \\
$\pi / 4$ & $\pi / 3.987$ & $\pi / 2.888$ & -1.5828 & -1.584 & 2007 \\
$\pi / 2$ & $\pi / 2$ & $\pi / 2$ & -2.0 & -2.0 & 2004 \\
\hline
\end{tabular}




\section{Figure captions}

Figure 1 Local magnetization structure in the vortex regime. $\left(\mathrm{R}=45 \mathrm{~nm} ; K_{1}=0\right.$; remanent state.). Top : projection of the local magnetic moment in the equatorial plane of the sphere, normal to vortex axis, $\hat{v}=\vec{M} /\|\vec{M}\|$. The length of the arrows is proportional to the norm of the projection of $\vec{m}(\vec{r}), \vec{m}_{p}(\vec{r})$. The central part of the vortex is clearly identified as the region where $\vec{m}_{p}(\vec{r})=$ 0 ; the direction of magnetization in this region coincides with the vortex direction, $\hat{v}$. Bottom : Local magnetization in the direction normal the equatorial plane of the vortex shown on top, along a diagonal of this last one. The vortex direction is shown as the large bold arrow.

Figure 2 Magnetization curve in the direction of the field. $R=45 \mathrm{~nm} ; K_{1}=0$.

Figure 3 Cylindrical components of the local magnetization profile at remanence. across the equatorial plane of the sphere $(z=0) . m_{v}$, solid line; $m_{\varphi}$ dashed line; $m_{\rho}$ dotted line. $\mathrm{R}=45 \mathrm{~nm}, K_{1}=0$.

Figure 1 Components $m_{v}$ (triangles), $m_{\varphi}$ (squares) and $m_{\rho}$ (circles) of the local magnetization profile in the vicinity of the coercive field before (solid symbols) and after (open symbols) reversal of the vortex core. $R=45 \mathrm{~nm}, K_{1}=0 . d=\rho \operatorname{sign}(y)$ where $\rho$ is radius in the equatorial plane $(z=0)$.

Figure 5 Magnetization curve parallel and normal to the field . $\hat{h}_{e x}=\hat{a}$ (solid line) or $\hat{v}$ (dashed line). $R=45 \mathrm{~nm}, K_{1}=3.10^{4} \mathrm{~J} / \mathrm{m}$.

Figure 6 Components $m_{v}$ (solid line), $m_{\varphi}$ (dashed line) and $m_{\rho}$ (dotted line) of the local magnetization profile relative to the vortex core across the $(x=0)$ plane. $R=45 \mathrm{~nm}, K_{1}=310^{4} \mathrm{~J} / \mathrm{m}^{3}, H_{e x}=$ $68 \mathrm{kA} / \mathrm{m}, \hat{a}=\hat{h}_{e x}=\hat{z}$ and $\hat{v}=\hat{x}$. The vortex is shifted along the $\hat{y}$ axis in the $y>0$ direction, by an amount $y_{c}=15.75 \mathrm{~nm}$, leading to a non symmetric range of variation for $d$. The location of the vortex core is indicated by the arrow.

Figure 0 Magnetization curve parallel and normal to the field for $K_{1}=310^{4} \mathrm{~J} / \mathrm{m}^{3}$ and different sizes. Magnetization in the direction of the field and : $R=26 \mathrm{~nm}$ (solid); $30 \mathrm{~nm}$ (short dash); $37 \mathrm{~nm}$ (long dash). Magnetization normal to the field : $R=30 \mathrm{~nm}$ (dot short dash); $37 \mathrm{~nm}$ (dot long dash). For $R=26 \mathrm{~nm}$, the magnetization normal to the field vanishes and the magnetization reversal occurs at a positive field since $R=26 \mathrm{~nm}$ enters in the range of particle sizes where the vortex direction is parallel to the easy axis, chosen as the direction for the field.

Figure 8 Variation of the interaction energy for 2 spheres at $r_{12}=4 R$ with the angular function $d_{112}$ characterizing the relative orientations, normalized by its maximum value, $E\left(d_{112}=2\right)-E(0)$. $R=35 \mathrm{~nm} ; K_{1}=0$.

Figure 9 Interaction energy per unit volume between two approaching spheres. $R=35 \mathrm{~nm} ; K_{1}=0$; $d_{112}=-2$. Open triangles: full calculation, from (12) (the thin line is a guide to the eye); solid line: equ.(17); dashed line: simple dipolar approximation, $u=0$.

Figure 10 Same as figure (9) for the interaction normalized by the value at $r_{12}=2.25 R$. dotted lines: result of equ.(17) with the values of $\alpha$ and $u$ fitted in order to improve the agreement with simulated results. Open triangles : $K_{1}=0, d_{112}=-2, R=35 \mathrm{~nm}$; open squares : $K_{1}=310^{4} \mathrm{~J} / \mathrm{m}^{3}, d_{112}$ $=-2, R=35 \mathrm{~nm}$; solid triangles : $K_{1}=310^{4} \mathrm{~J} / \mathrm{m}^{3}, d_{112}=-1, R=45 \mathrm{~nm}$; solid squares : $K_{1}=$ $310^{4} \mathrm{~J} / \mathrm{m}^{3}, d_{112}=-2, R=45 \mathrm{~nm}$. The different curves are shifted along the $r_{12}$ axis for clarity. 
Figure 1:
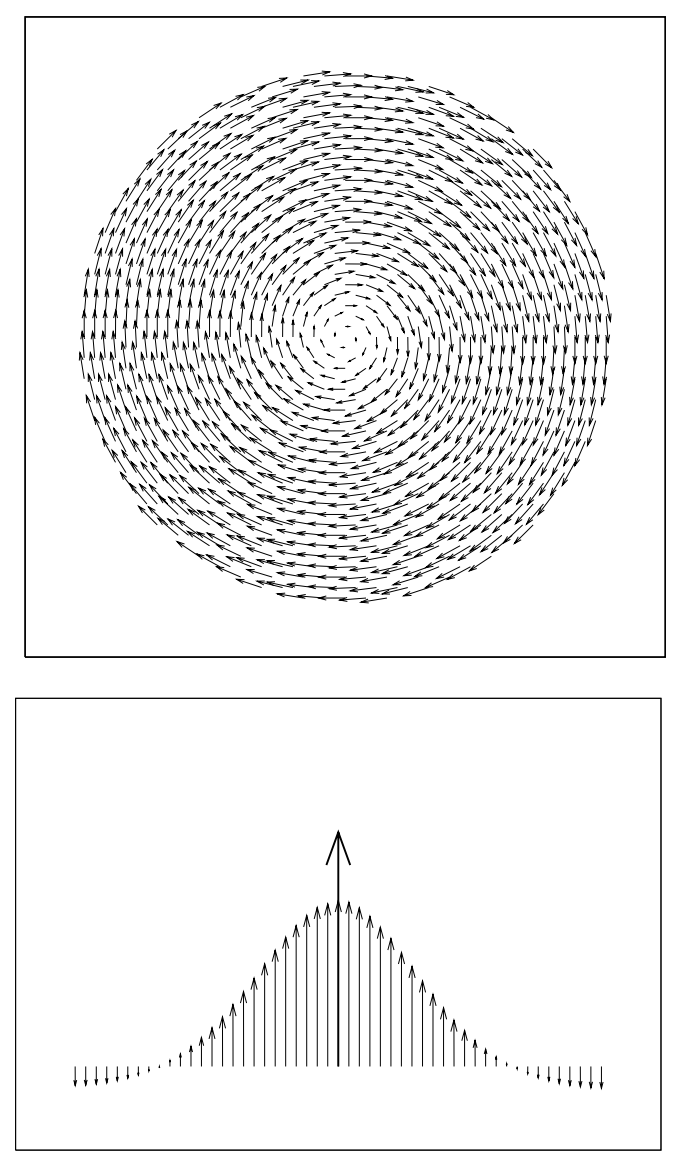

Figure 2:

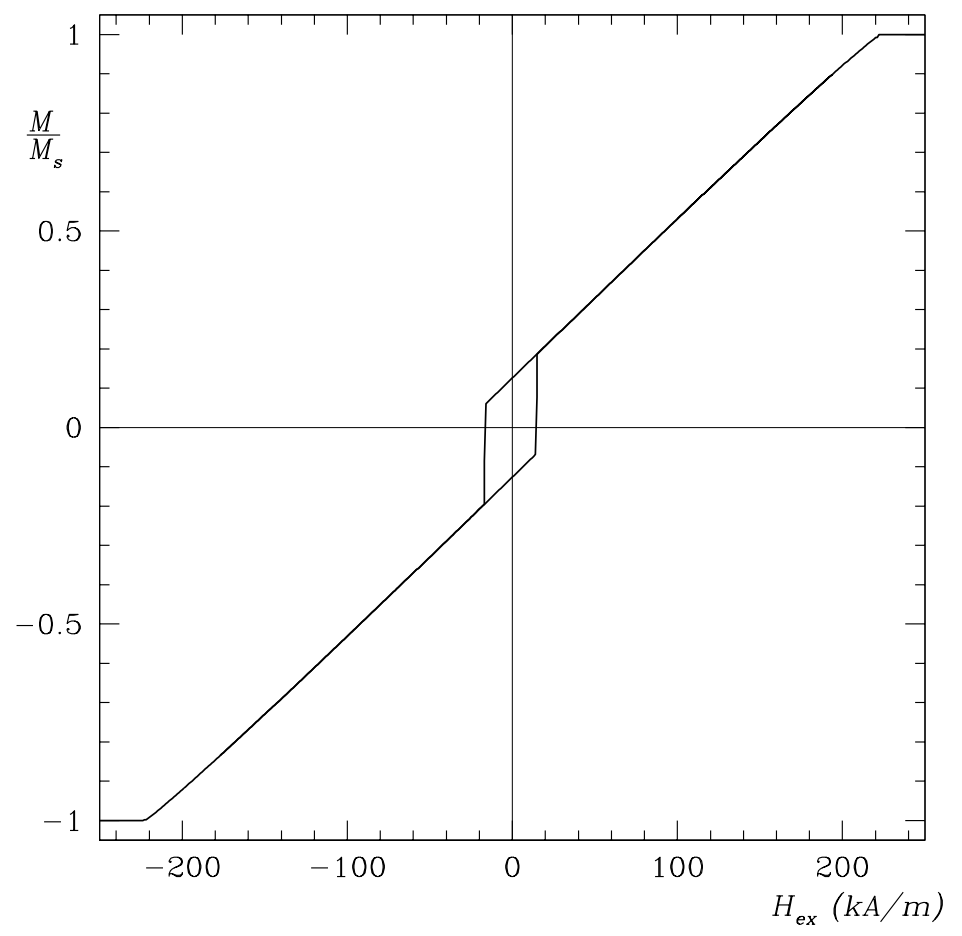


Figure 3:

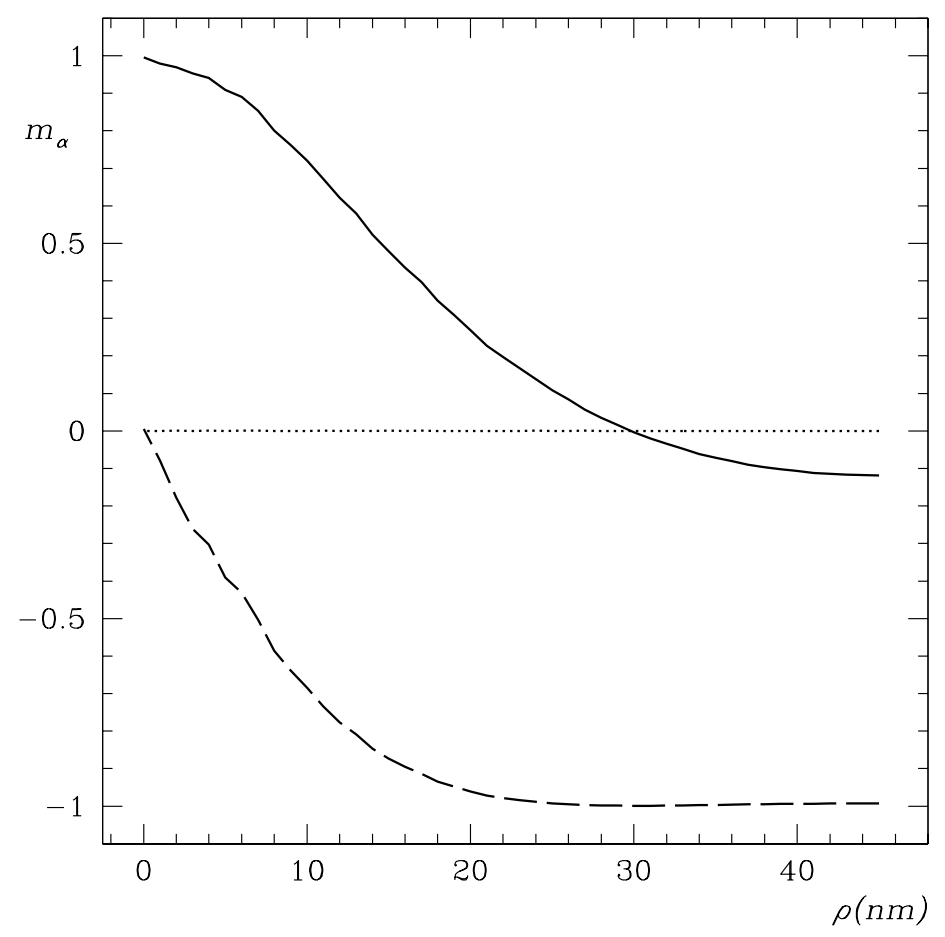

Figure 4:

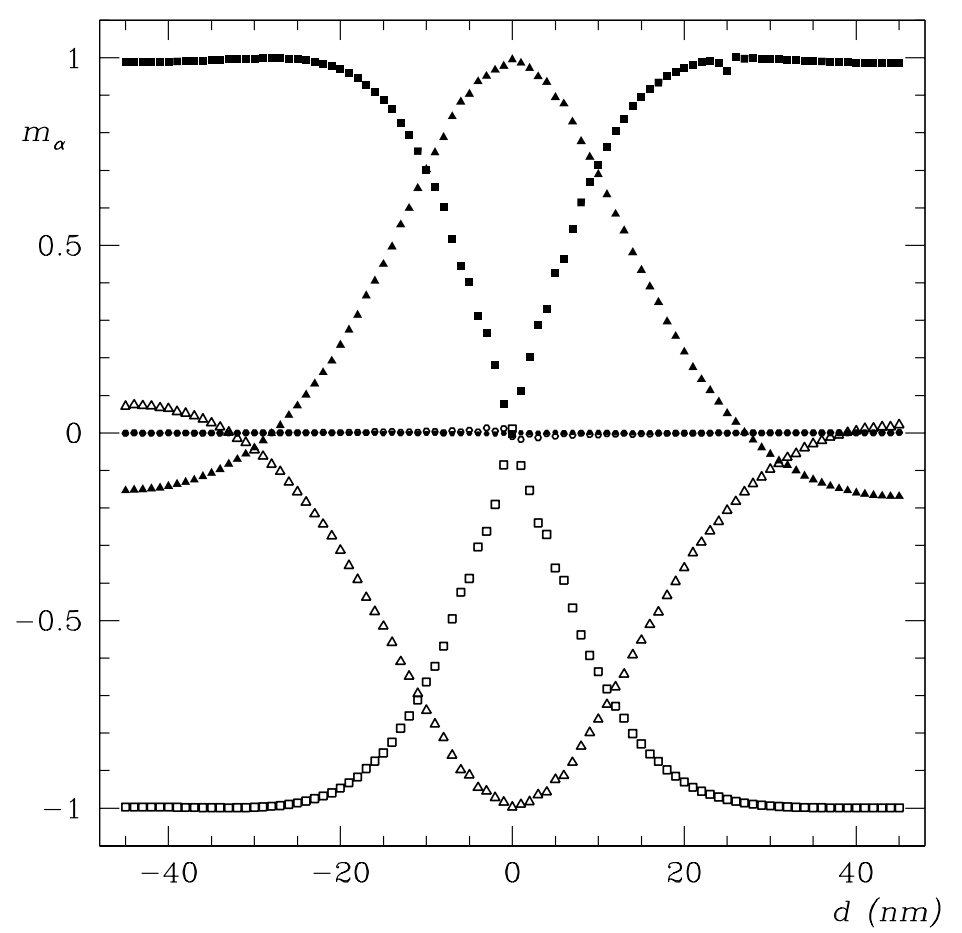


Figure 5:

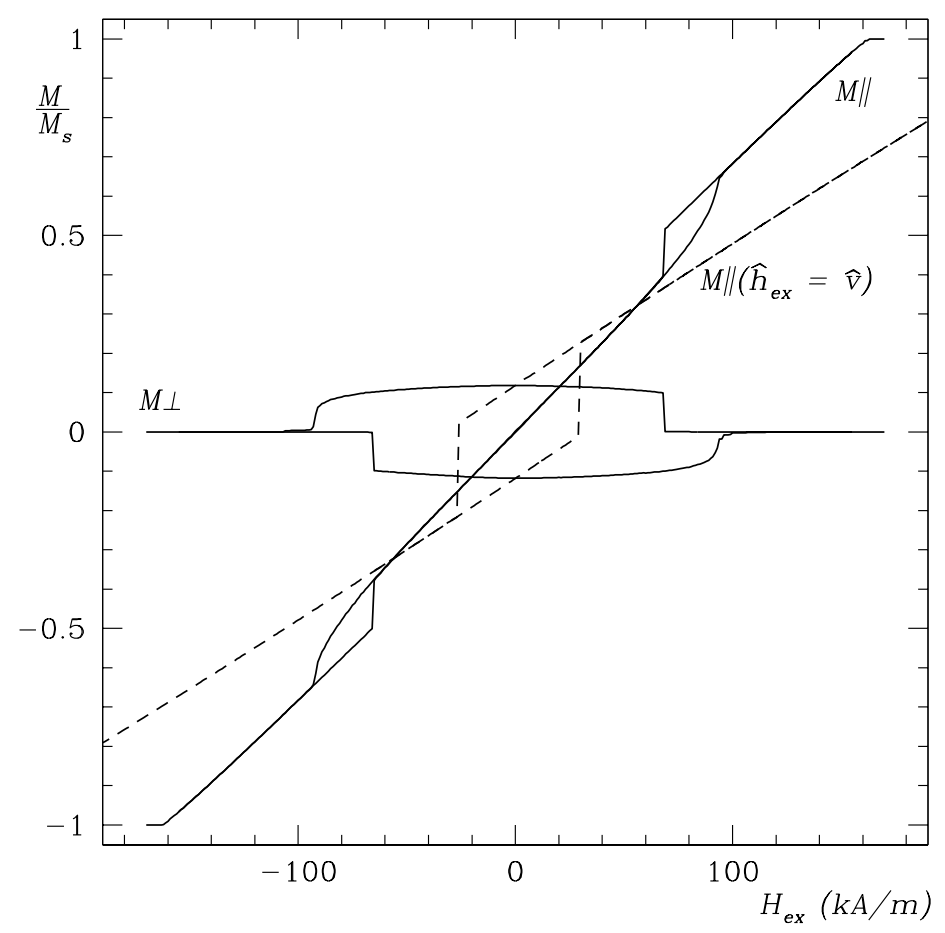

Figure 6:

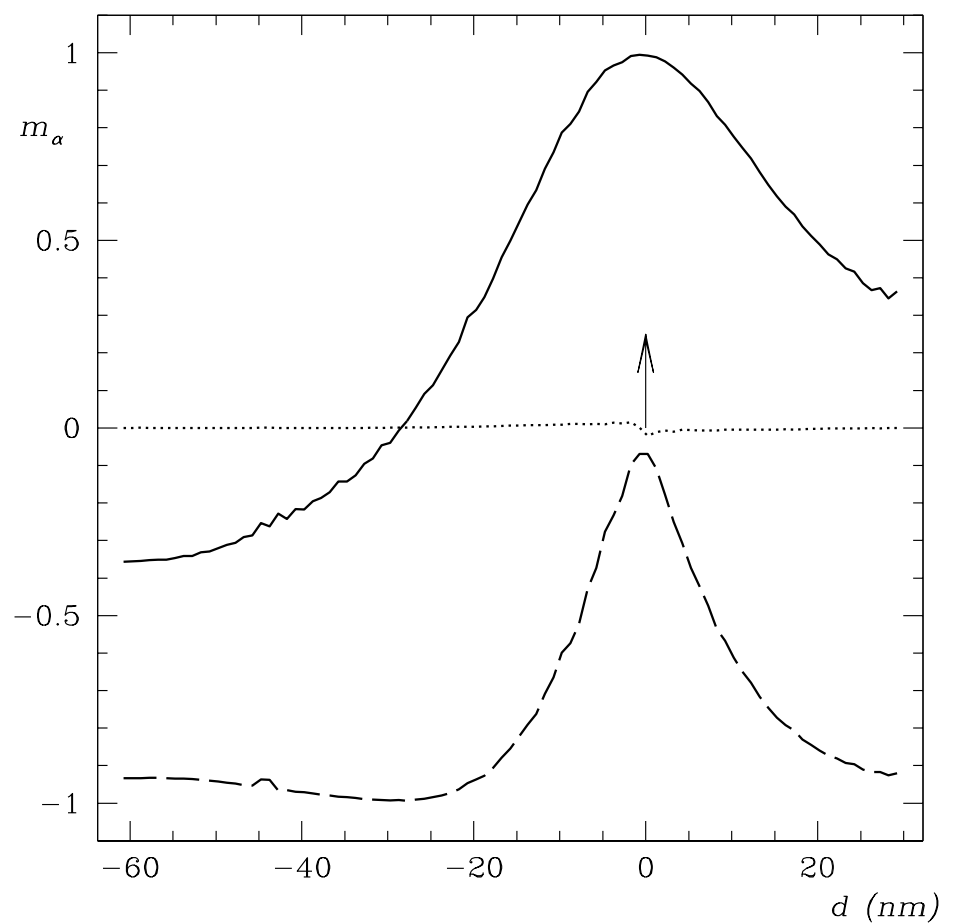


Figure 7:

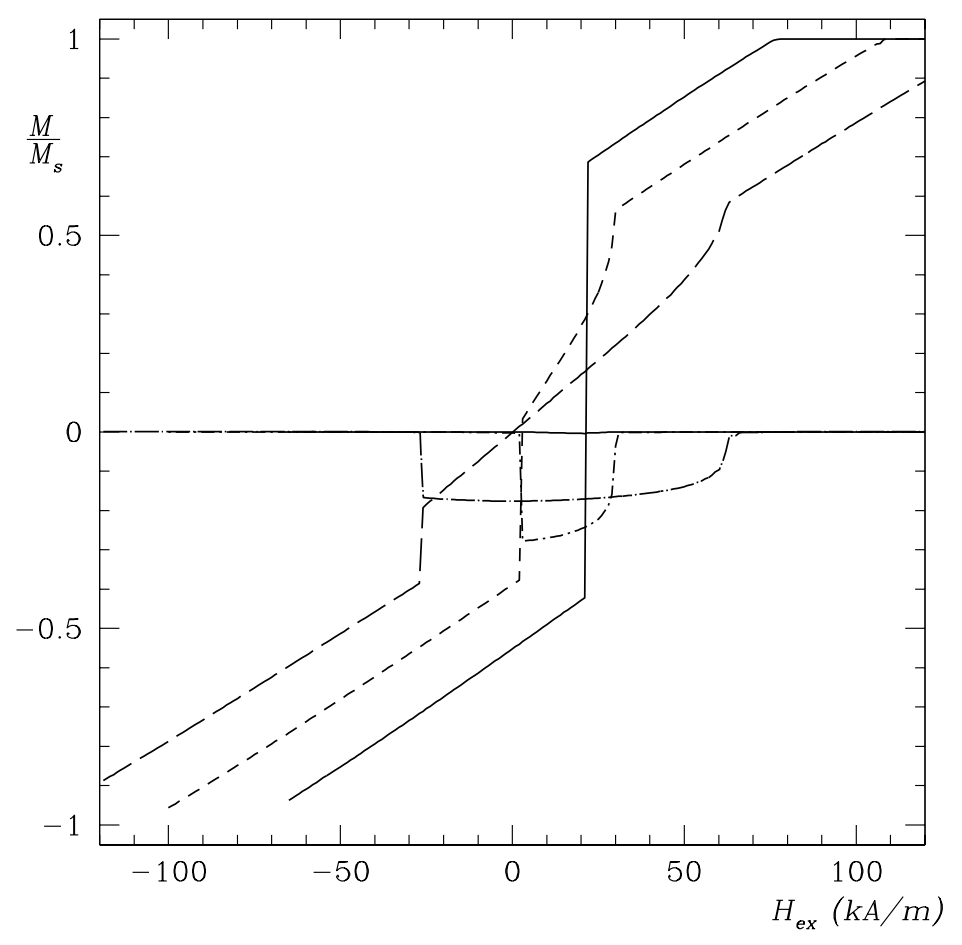

Figure 8:

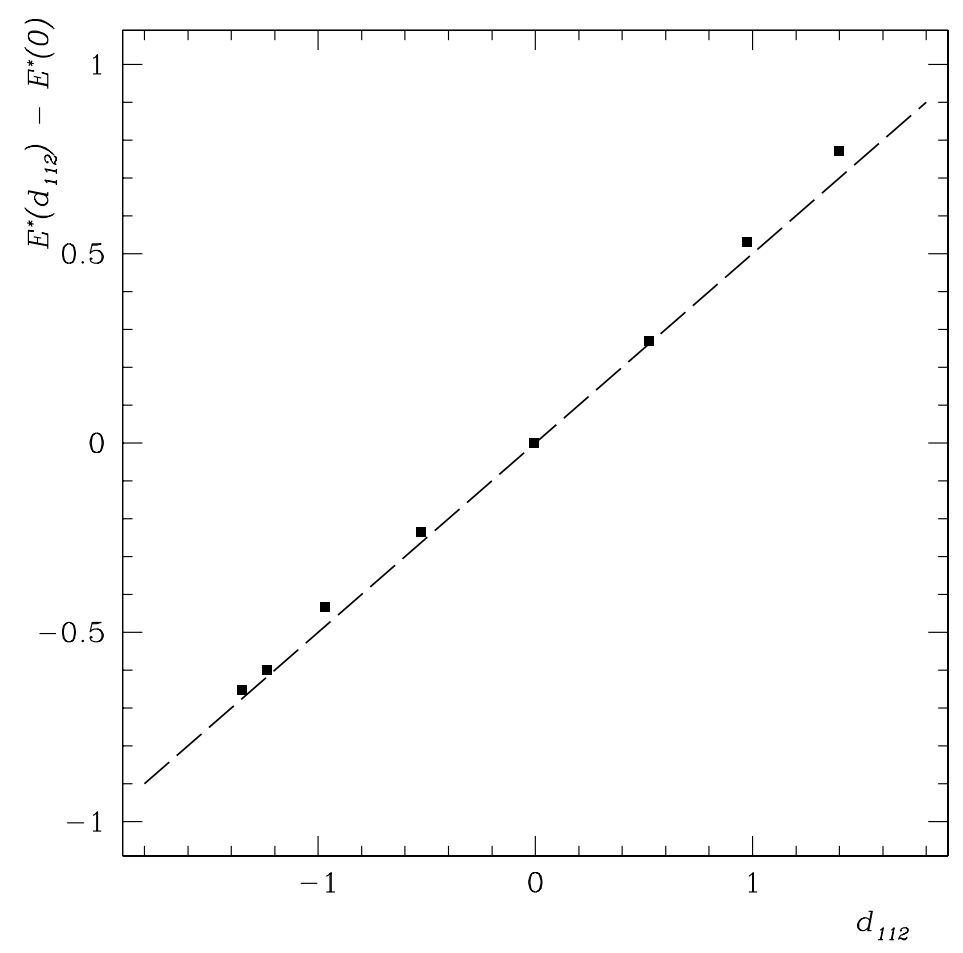


Figure 9:

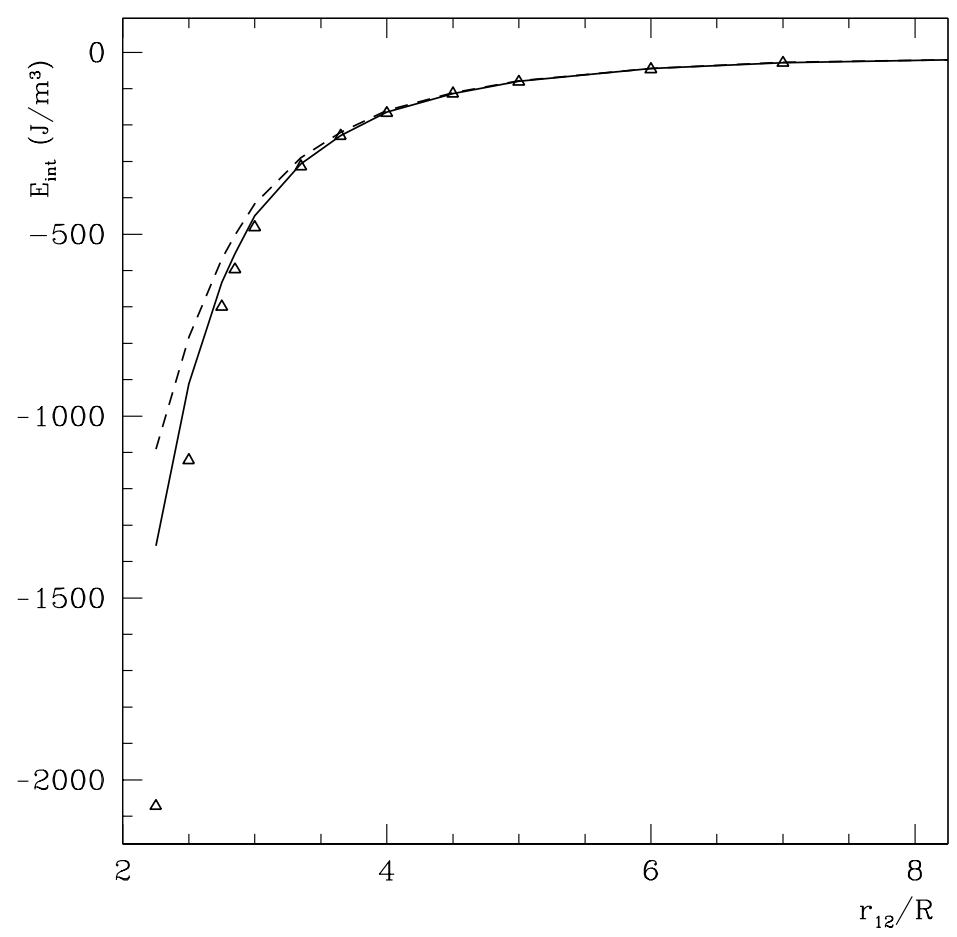

Figure 10:

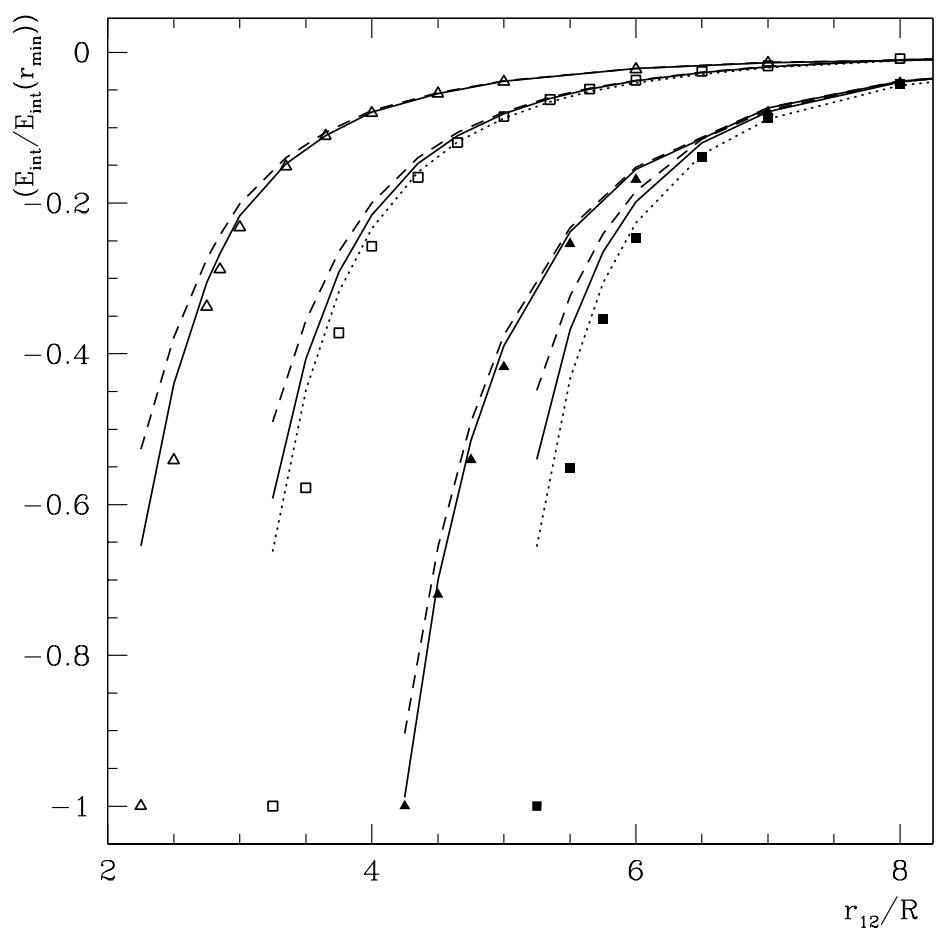

